\title{
The Localization Algorithm Based on Improved DV-Hop Algorithm
}

\author{
Jun-gang ZHENG ${ }^{1},{ }^{a}$, Li CHEN ${ }^{2, b}$ and Xing-hua XIA ${ }^{3, c}$ \\ ${ }^{1}$ School of Science, Shenyang Jian zhu University, Shenyang,110168,china \\ ${ }^{2}$ Architects\&Engineers, Shenyang Jian zhu University, Shenyang,110168,china \\ ${ }^{3}$ Information\&Control Engineering Faculty, Shenyang Jian zhu University, Shenyang,110168, \\ china

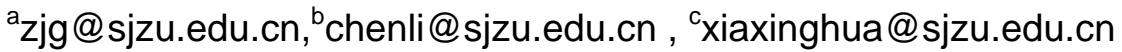

Keywords: DV-Hop; correction; localization; wireless sensor networks

\begin{abstract}
Node localization is the foundation of the wireless sensor networks. The traditional DVHop localization algorithm has been studied in this paper.In order to solve the problem of DV-Hop localization algorithm, an improved DV-Hop localization algorithm has been proposed. Which uses correction value reducing the measurement average hop distance error and selecting optimal anchor nodes by APIT algorithm. The simulation results demonstrate that,the improved localization algorithm(DV-SCA)has a better localization accuracy than the original DV-Hop algorithm.
\end{abstract}

\section{Introduction}

Wireless sensor network is an important technology,which is composed of a large number of sensor nodes.These sensor nodes have the capability of sensing ,computation ,and wireless communication. In many application of wireless sensor networks, it is needed to get the geographical localization of the sensor which detected an event.Localization information of sensor node is required to identify. Since node localization is precondition and foundation ,so it is one of the hotspots in current research[1][2].At present ,the localization algorithms of the sensor nodes are commonly classified into two categories: range-based and range-free,based on whether it is needed to measure the actual distances between the nodes or the distance is approximated based on the connectivity information. Range-based method use absolute point-to-point distance or angle information to estimate the location between neighboring sensors. Several ranging techniques are possible for range measurement, such as time of arrival (TOA), time difference of arrival(TDOA), angle of arrival(AOA), or the receive signal strength indicator(RSSI). Range-free techniques solution depends only on the contents of received messages, which does not estimate the distance or angle between the nodes. Typical range-free localization algorithms included Centroid , DV-Hop, Amorphous, MDS-MAP and APIT, and so on. In this paper ,we focus on the DV-Hop localization algorithm, the main advantage of DV-Hop algorithm are the low demand for hardware devices,convenient operation,high efficiency and low energy consumption,but the main localization error of DV-Hop localization algorithm is calculating the distance between nodes. DV-SCA algorithm has been proposed, which uses correction value reducing the measurement average hop distance error and selecting optimal anchor nodes by APIT algorithm. Simulation results demonstrate that DV-SCA algorithm is more higher than DV-Hop algorithm.

\section{DV-Hop localization algorithm}

Niculescu and Nath have proposed the DV-Hop algorithm, which is a distributed, hop by hop positioning algorithm[3][4][5]. The basic idea is: calculating the distance between the unknown nodes and anchor nodes, which based on the average per hop-length and hops between unknown nodes and anchor nodes. When unknown node receive the distance of three or more anchor nodes. Unknown nodes can calculate on position information according to trilateration method. The specific algorithm is as follows: 
- An anchor node broadcast the message to the entire network, which includes anchor node' s ID, coordinates and hops, using flood control method. The anchor node' s hops has been received by all nodes.

- According to the received messages ,the anchor nodes calculate the average hop-length and broadcast its hop-length to the entire network. Unknown nodes receive hop-length information. Unknown nodes calculate the distance to anchor nodes based on the product of hop-length and hops to the anchor nodes.

-When unknown node receive the Eulidean distance of three or more anchor nodes. Unknown nodes can make out own position information according to Trilateration or Maximum Likelihood Estimation. (average hop-distance) of anchor nodes $\left(\mathrm{X}_{\mathrm{j}}, \mathrm{Y}_{\mathrm{j}}\right)$, which can been shown by Formula (1) :

$$
\text { HopSize }_{i}=\frac{\sum \sqrt{\left(X_{i}-X_{J}\right)^{2}+\left(Y_{i}-Y_{J}\right)^{2}}}{\sum h_{i j}}(i \neq j)
$$

Where $\left(x_{j}, y_{j}\right)$,is the localization of the anchor $j, h_{i j}$ is the number of hops between the anchor $i$ and the anchor $\mathrm{j}$.

The unknown node distance estimation from anchor nodes is given by:

$$
\left\{\begin{array}{c}
\left(x_{1}-x\right)^{2}+\left(y_{1}-y\right)^{2}=d_{1}^{2} \\
\left(x_{2}-x\right)^{2}+\left(y_{2}-y\right)^{2}=d_{2}^{2} \\
\cdots \\
\left(x_{n}-x\right)^{2}+\left(y_{n}-y\right)^{2}=d_{n}^{2}
\end{array}\right.
$$

Equation(2) can be written in matrix from $A X=b$ by subtracting the last equation from previous n-1:

$$
\begin{aligned}
& A=\left[\begin{array}{c}
2\left(x_{1}-x_{n}\right) 2\left(y_{1}-y_{n}\right) \\
2\left(x_{2}-x_{n}\right) 2\left(y_{2}-y_{n}\right) \\
\cdots \\
2\left(x_{n-1}-x_{n}\right) 2\left(y_{n-1}-y_{n}\right)
\end{array}\right] \\
& b=\left[\begin{array}{c}
x_{1}^{2}-x_{n}^{2}+y_{1}^{2}-y_{n}^{2}+d_{n}^{2}-d_{1}^{2} \\
x_{2}^{2}-x_{n}^{2}+y_{2}^{2}-y_{n}^{2}+d_{n}^{2}-d_{2}^{2} \\
\cdots \\
x_{n-1}^{2}-x_{n}^{2}+y_{n-1}^{2}-y_{n}^{2}+d_{n}^{2}-d_{n-1}^{2}
\end{array}\right] \\
& X=\left[\begin{array}{l}
\mathrm{x} \\
y
\end{array}\right]
\end{aligned}
$$

The localization of unknown node is obtained by applying least square method,which can be expressed as:

$$
\mathrm{X}=\left(\mathrm{A}^{\mathrm{T}} \mathrm{A}\right)^{-1} \mathrm{~A}^{\mathrm{T}} \mathrm{b}
$$

The advantages of DV-Hop algorithm are simple, convenient operation, high efficiency, low hardware requirements and low energy consumption[6]. DV-Hop algorithm supposes that the product of the minimum hop count value and the average hop distance is the estimated distance between unknown nodes and the anchor nodes. Which assumes that the minimum hop path between nodes is similar to a straight line, but in practical applications, this is not always feasible. When the estimated value and the actual value of the average hop distance have deviation, localization error will be increased. The error of DV-Hop algorithm is shown in Figure1.

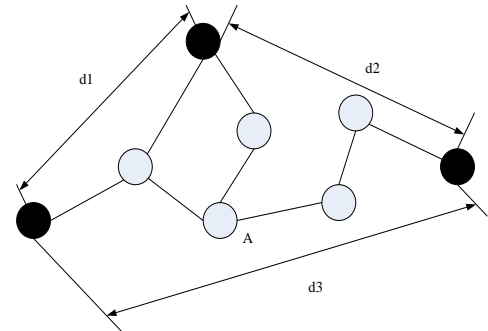

Fig.1 the error of DV-Hop algorithm

In figure1, $\mathrm{A}$ is the unknown node that needs to be localizated.The three black point are the 
anchor nodes.These anchor nodes know the distance from each other.From figure1,it can be seen that the distance between node A to the left anchor node is not equal to the distance between node A to middle anchor node.However,in the traditional DV-Hop localization process, the hop between node A to the left anchor node is 2,and the hop between node A to middle anchor node is also 2.

\section{The improved DV-hop algorithm}

Through analyzing the DV-hop algorithm,the main impact factors in nodes localization accuracy is the calculation of the average hop distance. In this paper,we make some improvements in the calculation of average hop distance and select anchor nodes.

When the average hop estimate distance is far away the actual hop distance,the estimate distance is far away the actual distance between the anchor nodes and unknown nodes. When the localization of unknown node is equated by applying the estimated distance,the localization precise is low. In order to get actual average hop distance,the error correction value $e_{i j}$ has been adopted[7]. The corrective steps are as follows:

$\mathrm{D}_{\mathrm{ij}}^{\text {true }}$ is the true distance between the anchor node $\mathrm{i}$ and anchor node $\mathrm{j}$.

$D_{i j}$ is estimate distance between the anchor node $i$ and anchor node $j$.

$E_{\mathrm{ij}}$ is the total distance estimation error between the anchor node $\mathrm{i}$ and anchor node $\mathrm{j}$.

$\mathrm{C}_{\mathrm{m}}^{2}$ is the number of total distance estimation error in localization region with $\mathrm{m}$ anchor nodes. $\mathrm{e}_{\mathrm{ij}}$ is the error correction value.

HopSize' $\mathrm{i}_{\mathrm{i}}^{\prime}$ is correction average hop distance between anchor node $\mathrm{i}$ and anchor node $\mathrm{j}$.

$$
\begin{aligned}
& D_{i j}^{\text {true }}=\sqrt{\left(x_{i}-x_{j}\right)^{2}-\left(y_{i}-y_{j}\right)^{2}} \\
& D_{i j}=H_{o p S i z e} \times h_{i j} \\
& E_{i j}=\sum_{j \neq i}\left(D_{i j}^{\text {true }}-D_{i j}\right) \\
& e_{i j}=\frac{E_{i j}}{C_{m}^{2}}=\frac{1}{C_{m}^{2}} \sum_{j \neq i}\left(D_{i j}^{\text {true }}-D_{i j}\right)
\end{aligned}
$$

Finally, the anchor node broadcast the correction average hop distance to the networks.

The anchor nodes selection is based on the APIT algorithm[8]. The steps are as follows:

Step1: From $n$ anchor nodes, select combinations of $m$ anchors, which meet the formula (12).In formula(11), $d_{1}, d_{2}, d_{3}$ are respectively the distance between anchors, which are calculated by the anchor node coordinates. Which is shown in Figure2.

$$
\left\{\begin{array}{l}
d_{1}+d_{2}>d_{3} \\
d_{2}+d_{3}>d_{1} \\
d_{1}+d_{3}>d_{2}
\end{array}\right.
$$

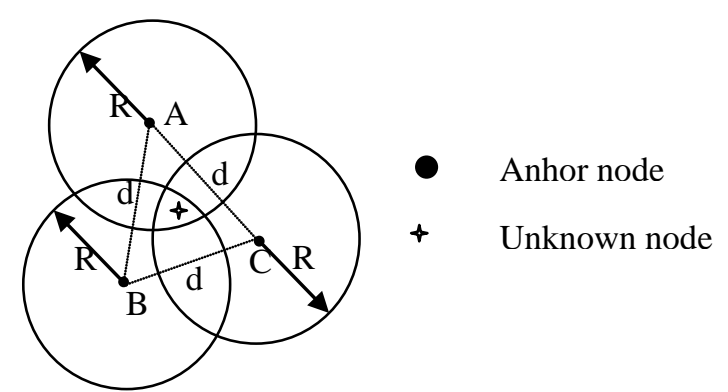

Fig.2 optimal situations

Step2:Selecting $\mathrm{k}$ node combinations of $\mathrm{m}$ anchors.Anchor nodes in combination can pose an acute triangle.The distance between anchor nodes meeting formual(13)

$$
\left\{\begin{array}{l}
\mathrm{d}_{1}^{2}+\mathrm{d}_{2}^{2}<\mathrm{d}_{3}^{3} \\
\mathrm{~d}_{3}^{2}+\mathrm{d}_{2}^{2}<\mathrm{d}_{1}^{2} \\
\mathrm{~d}_{1}^{2}+\mathrm{d}_{3}^{2}<\mathrm{d}_{2}^{2}
\end{array}\right.
$$


Step3:Determining whether the unknown node in an acute triangle by APIT algorithm,the acute triangle comes from step2.

\section{DV-SCA algorithm implementation}

Firstly, determining the hops $h_{i j}$ between nodes(i,j is any two nodes in the network),secondly, calculating the correction average hop distance by the method proposed in this paper, finally, selecting appropriate anchor nodes by the method proposed in this paper, calculating the distance between nodes, calculating the coordination of unknown nodes with Trilateration.

In order to verify the effectiveness and availability of the algorithm ,the simulation of the algorithm has been finished. The main parameters of the simulation network model is as follows: Nodes have been distributed randomly in $100 \mathrm{~m} \times 100 \mathrm{~m}$ square area , The radio range is general the same for every node. The algorithm is tested through RMES, the formula is shown in (14)

$\operatorname{RMSE}=\sqrt{\frac{\sum_{\mathrm{i}=1}^{\mathrm{N}}\left(\widehat{\mathrm{x}}_{1}-\mathrm{x}_{\mathrm{i}}\right)^{2}+\left(\widehat{\mathrm{y}}_{\mathrm{i}}-\mathrm{y}_{\mathrm{i}}\right)^{2}}{\mathrm{n}}}$

$\left(\widehat{x}_{1}, \widehat{y}_{1}\right)$ are unknown nodes coordinates, $\left(\mathrm{x}_{\mathrm{i}}, \mathrm{y}_{\mathrm{i}}\right)$ are real coordinates, $\mathrm{N}$ is the number of unknown nodes.

The result of simulation is shown in Fig.3 Fig.4 and Fig.5.

Figure.3 show that under different communication radius ,the measurement error of DV-SCA algorithm in one hop is low the DV-HOP algorithm. Compared with DV-HOP ,the reducing error value is about $56 \%$.

Figure. 4 show that under the same mumber anchor and different communication radius, the localization error of DV-SCA algorithm is lower than DV-HOP algorithm , the value is about $20 \%$.

Figure.5 show that under different communication radius, the localization accuracy of DV-SCA algorithm is higher than DV-HOP algorithm ,the value is about $10 \%$.

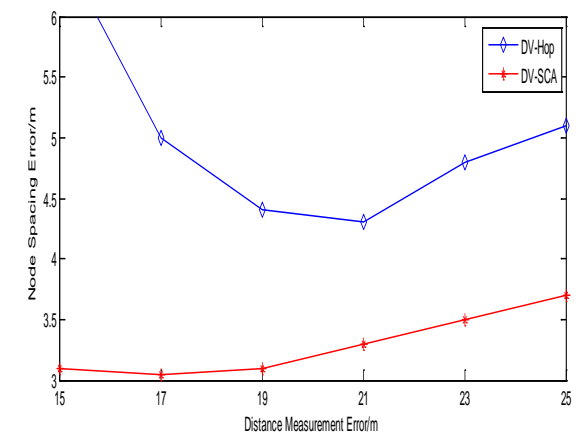

Fig.3 Meanhop-distance vs. communication radius

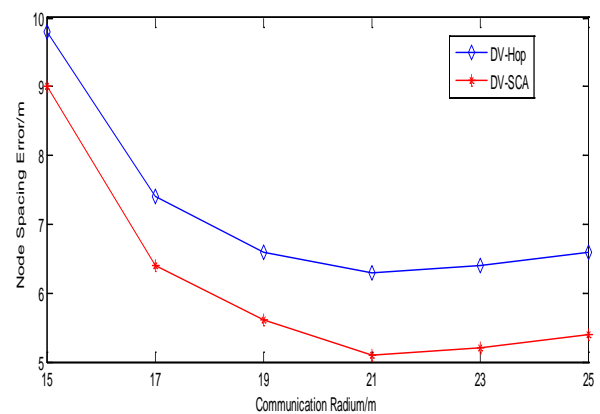

Fig.4 Error under different coordinate calculation method 


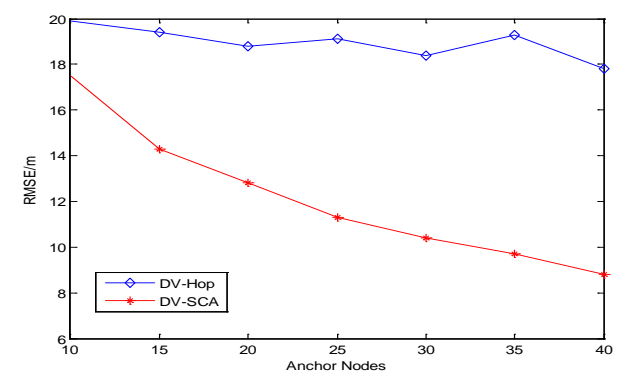

Fig.5 RMSE in terms of anchor node density

\section{Conclusion}

In this paper, we proposed an improved localization algorithm according to the error of DV-Hop algorithm.DV-SCA localization algorithm mainly uses correction value reducing the measurement average hop distance and selecting optimal anchor nodes by APIT algorithm. Simulation shown that this algorithm can meet most applications in wireless sensor networks.

\section{Acknowledgement}

This research was financially supported by the education department of Liaoning province. The grant number is L2015455

\section{References}

[1] I.F. Akyildiz, W.Su, Y.Sankarasubramaniam, and E.Cayirci. Wireless sensor network: a survey[J].Computer Networks(Elesvier), 2002 38(4) 393-422.

[2]Q.Yu,S.Y.Sun,B.G.Xu and S.J.Chen.Node localization in wireless sensor networks based on improved particle swarm optimization[J].Journal of Computer Application, 2015 32(6) 15191522,1544 .

[3]S.Ergut,R.R.Rao,O.Dural,and Z.Sahinoglu.Localization via TDOA in UWB sensor network using neural networks[C]. in Proc.IEEE International Conference onCommunications,2008.23982403.

[4]Y.Sun Lee,J.Woo Park,and L.Barolli.A localization algorithm based on AOA for ad-hoc sensor networks[J].Mobile Information Systems,2012.61-72.

[5]K.Benkic,M.Malajner,P.Planinsic,and Z.Cucej.Using RSSI value for distance estimation in wireless sensor networks based on ZigBee[C].in Proc.15 ${ }^{\text {th }}$ International Conference on Systems,Signals and Image Processing,2008.303-306.

[6]Dan Zhang,Zhiyi Fang,Yue Wang and so on.A New Improved Range Free Algorithm Based on DV-Hop in Wireless Sensor Network[J].Journal of Communications, 2015 10(8) 598-595.

[7]Zhenqin Di,Shuli Ma,and so on.A modified method for obtaining correction value of unknown nodes on DV-Hop algorithm[J].Communications technologhy, 2016 49(3) 329-334.

[8]He,T.,Huang,C.,and so on.Rang-free localization schemes for large scale sensor networks[C],In proceedings of the $9^{\text {th }}$ annual indternational conference on mobile computing networking.2003 8195. 